\title{
MICRO-MRI VELOCIMETRY IN MICROCHANNEL NETWORKS
}

\author{
L. Guy Raguin, Dimitris C. Karampinos, and Sharon L. Honecker \\ Mechanical \& Industrial Engineering Dept., University of Illinois \\ Urbana, Illinois USA \\ John G. Georgiadis \\ Mechanical \& Industrial Engineering Dept. and the Beckman Institute, University of Illinois \\ Urbana, Illinois USA
}

\begin{abstract}
We report here the first use of microscopic Magnetic Resonance Imaging (micro-MRI) velocimetry to quantify both the interstitial topology and the pressure-driven flow of water through an engineered microchannel network made from polydimethylsiloxane (PDMS) using a standard volume radiofrequency coil (7.6 cm ID). The multilayered PDMS microchannel network consists of two identical non-communicating capillary networks, each featuring 1.9-mm-deep and 0.9-mm-wide staggered channels forming a $10 \times 8$ periodic array. A multi-slice spin-echo sequence with $117 \mu \mathrm{m} \times 156 \mu \mathrm{m}$ in-plane resolution is employed to reconstruct the interstitial geometry of the network. The superiority of phase contrast micro-MRI velocimetry is demonstrated first in simpler microchannels, and then used to extract the two-dimensional velocity field in the complex microchannel network. The micro-MRI velocity measurements have a spatial resolution of $195 \mu \mathrm{m} \times 254 \mu \mathrm{m}$ and are accurate in terms of overall mass conservation to within $3 \%$ of the imposed flow rate.
\end{abstract}

\section{INTRODUCTION}

Given the complexity of the underlying physicochemical phenomena, the fabrication of microfluidic systems [1-3] needs be coordinated with the in-situ characterization of the function of their components. The majority of quantitative methods used to probe flow in microchannels rely on visible light, such as particle tracking techniques (e.g. micro-PIV [4]), infrared light (e.g. optical coherence tomography [5], or infrared PIV [6]). Despite the excellent spatial resolution, the ability to measure fluid velocity in topologically complex geometries remains limited, since such methods rely on optical penetration, matching the index of refraction in the field of view, and the introduction of tracer particles. Refraction index matching is impossible for non-planar or multiphase fluidic systems such as those found in endothelialized microvascular networks [3]. The seeding of particles in flows where multiple force fields are present, such as in electrokinetic flows, presents an additional challenge since these particles tend to become charged and diverge from streamlines, producing the so-called electrophoretic drift [7]). In addition, the extraction of the full velocity field in complex microflows via PIV requires high numerical aperture optics, which limits the depth of focus [8] and consequently decreases the optical penetration depth.

We propose the use of Magnetic Resonance Imaging (MRI) to characterize complex microfluidic devices fabricated with polymeric materials. There have been earlier successful attempts to measure velocity in single microchannels with MRI [9-10] but they required specialized radiofrequency (RF) micro-coils, wrapped around the individual microchannel. The present work marks the first time that MRI employing standard volume RF coils, not integrated to the sample, is used to characterize singlephase flow through complex microchannel networks.

The closest systems to the microchannel network are 3-D lattices of packed particles with the interstitial space fully saturated with a liquid. One of the first systematic applications of MRI to characterize pressure-driven flow through such systems was reported by Shattuck et al. [11-12]. A number of average flow velocities comparisons between MRI velocimetry results and flowmeter measurements have been since reported for packed beds constructed by packing randomly spherical beads in a tube. Using pulsed-field-gradient MRI sequences and glass sphere beds, Deurer et al. [13] reported that MRI overpredicts the average velocity by $50 \%$, while Ren et al. [14] reported that MRI overpredicts by $27 \%$ for 0.6 -mm-spheres. Using phase-encoding MRI velocimetry, Bijeljic et al. [15] reported 2-D measurements in Stokes flow across aligned and staggered rows of millimeter-sized cylinders confined inside a Hele-Shaw cell but only qualitative statements about the interstitial velocity field were made. Moser [16] used phase contrast MRI to measure axial interstitial velocities in a packed bed made by randomly packing 3.175-mmdiameter spherical acrylic beads. Overall, the computed mass flow rate in each slice agreed with the flowmeter reading to within $5.3 \%$ $-6.4 \%$ depending on the flowrate.

Since there are large discrepancies in the literature in terms of the accuracy of the various MRI velocimetry protocols, the search for the best protocol is conducted in simple microchannels, before we turn our attention to the complex microchannel network.

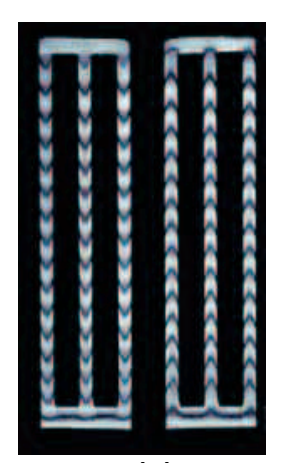

(a)

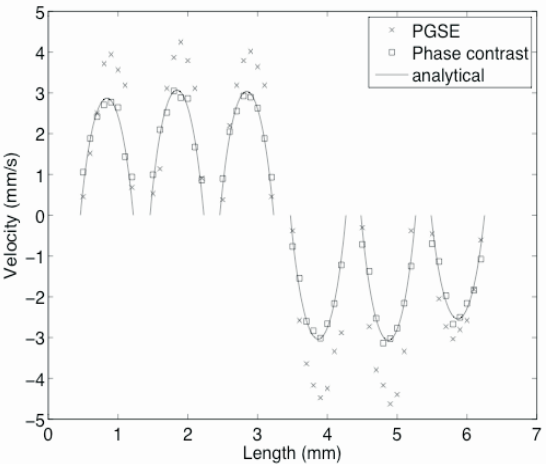

(b)
Figure 1. Comparison of axial velocity profiles obtained from three micro-MRI velocimetry protocols. (a) Spin-tagging spin-echo marks flow in the simpler microchannel network; (b) pulsedgradient spin-echo (PGSE) and phase contrast spin echo, in six parallel microchannels, for which an analytical solution is known.

\section{MRI VELOCIMETRY PROTOCOLS}

Using stereolithography, a simple microchannel network was built featuring six parallel channels, each with a rectangular crosssection of approx. $750 \mu \mathrm{m} \times 850 \mu \mathrm{m}$. Water doped with copper 
sulfate $(\mathrm{T} 1 / \mathrm{T} 2=0.25 / 0.22 \mathrm{~s})$ flows in one direction inside three microchannels, and returns through the other three, cf. Figure 1(a). The fluid interconnects between the two sets of co-current flow channels are outside the slice shown in Figure 1(a). An analytical solution is available for the velocity profile of fully-developed channel flow [17]. MRI experiments were conducted using a widebore 14.1 Tesla vertical imaging system (Oxford Instruments, Abingdon, UK) with a gradient set (Resonance Research, Billerica, MA) capable of up to $90 \mathrm{G} / \mathrm{cm}$ in $0.1 \mathrm{~ms}$, resulting in a clear bore of $46 \mathrm{~mm}$. Three MRI velocimetry protocols are investigated: phase contrast spin-echo (PC-MRI [18]), pulsed-gradient spinecho (PGSE [19]), and spin-tagging spin-echo (STSE [20]). For all three protocols, we used a field of view $(F O V)$ of $1.6 \mathrm{~cm} \times 2 \mathrm{~cm}$ with uniform in-plane resolution of $100 \mu \mathrm{m}$, and slice thickness of $1.2 \mathrm{~mm}$.

Velocity fields were acquired flows with $\operatorname{Re}<5$. (Typically, Reynolds numbers are based on the channel width.) Figure 1(a) shows the STSE results $(\operatorname{Re}=4)$, which provides a qualitative image of the flow. Figure 1(b) compares the velocity profiles inside the six channels measured using PGSE and PC-MRI $(\mathrm{Re}=$ 3.3) to the analytical solution averaged over the depth of the channels. Our preliminary results [21] indicate that for MRI velocimetry in microchannels, STSE provides good qualitative results but lacks in resolution, PGSE produces biased estimates because the velocity distribution is not symmetrical for the voxels at low resolution compared to the channel size, while the velocity measurements via PC-MRI provide the best agreement with the analytical solution.
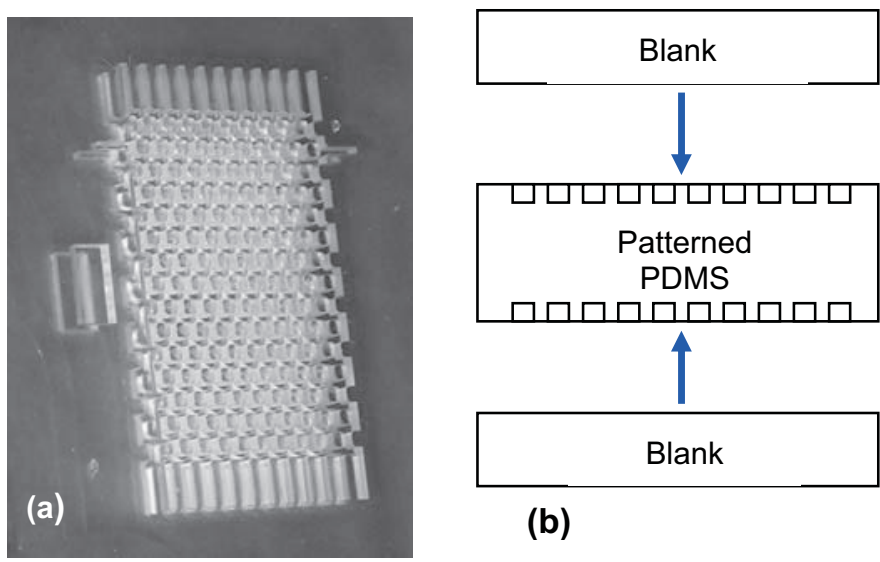

(b)

Figure 2. Fabrication of the microchannel network: (a) Patterned central PDMS layer with capillary bed relief on both sides. (b)Flat layers bonded to form the microchannel network.

\section{MICROCHANNEL NETWORK}

Figure 2 describes the fabrication of the test section consisting of two identical non-communicating capillary networks, each with two side ports to perform future infusion studies. Each capillary network features inter-connecting staggered channels forming a $10 \times 8$ periodic array. The PDMS layers were fabricated using variations of replica molding which involves pouring the PDMS prepolymer mixture (Sylgard 184, Dow Corning) over a master and curing it at $75^{\circ} \mathrm{C}$ for 12 hours. A novel two-sided mold was used to create the central patterned layer and the two blank layers were fabricated to a precise thickness using the molding process proposed by Jo et al. [22]. The two blank PDMS layers are bonded to the patterned PDMS layer after reactive ion etching pretreatment and the microchannels are connected via mm-sized tubes to fluid supply plenums fed by syringe pumps. Ultrasound waves were used to eliminate air bubbles from the network.

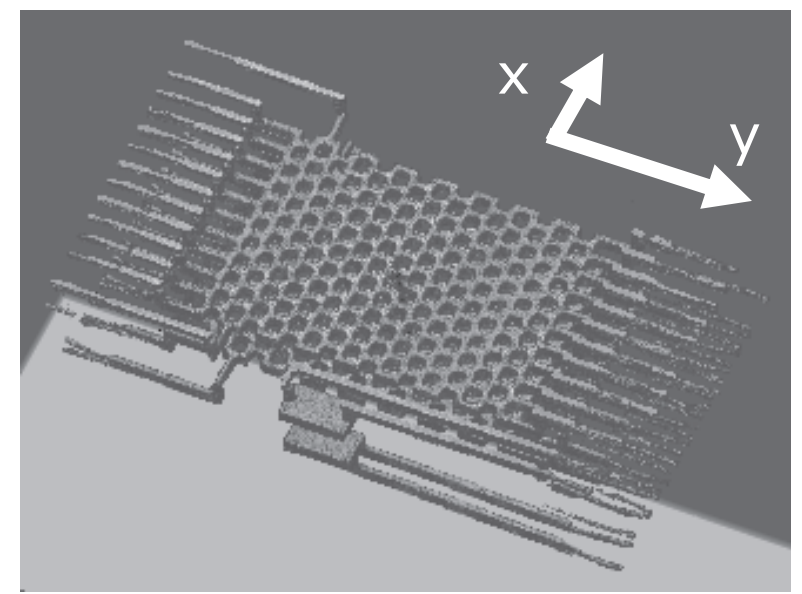

Figure 3. Volumetric rendering of the water-filled spaces in the microchannel network via spin density MRI protocol. The signal corresponds to the local water density and therefore delineates the interstitial space occupied by water. The mean flow is along the $y$ axis. The $x$-axis corresponds to the phase-encoding, and the y-axis to the read-out directions for MRI.

Figure 3 illustrates the use of micro-MRI to determine the critical (internal) dimensions of the microfluidic network. MRI experiments were conducted using a Varian 4.7 Tesla horizontal imaging spectrometer with Oxford ASG-26 gradients capable of up to $6.5 \mathrm{G} / \mathrm{cm}$ in $0.5 \mathrm{~ms}$. The spatial resolution of the velocity measurement is $195 \mu \mathrm{m} \times 254 \mu \mathrm{m}$. This is the result of a compromise between imaging time and field of view $(5 \mathrm{~cm} \times 6.5$ $\mathrm{cm})$, given the RF coil inner diameter $(7.6 \mathrm{~cm})$ and maximum gradient strength available. For comparison, Zhang and Webb [10] achieved a resolution of $15.6 \mu \mathrm{m} \times 15.6 \mu \mathrm{m}$ with their $3.2-\mathrm{mm}$ long RF coil wrapped around a single microchannel ( $800 \mu \mathrm{m}$ OD).

\section{MRI VELOCIMETRY}

The microchannel network was fully flooded with a aqueous solution of copper sulfate to lower the relaxation time T1 to $1 \mathrm{~s}$ (instead of $3 \mathrm{~s}$ for deionized water), hence allowing for shorter repetition times (TR) and reducing the overall image acquisition time. Two sets of micro-MRI phase contrast velocimetry experiments were performed: the first to illustrate the advantages of correcting for partial volume effects, and the second to assess the quality of the velocity measurements by checking mass conservation along the mean flow direction of the microchannel network.

A 2-D velocity data set was acquired for a Reynolds number $\mathrm{Re}=1$ (volumetric flow rate $\mathrm{Q}=54.8 \mathrm{ml} / \mathrm{hr}$, average axial velocity in the axial channels of the network Uavg $=0.8 \mathrm{~mm} / \mathrm{s}$, with Re based on the single channel hydraulic diameter and Uavg) with a high spatial resolution: 256 data points are collected in both read-out and phase-encoding direction for a $5 \mathrm{~cm} \times 6.5 \mathrm{~cm}$ FOV and a $3.25-\mathrm{mm}$ slice thickness. This yields a spatial resolution of $195 \mu \mathrm{m}$ in the phase-encoding direction (x-axis) and $254 \mu \mathrm{m}$ in the read-out direction (y-axis). The data matrix is then zero-padded to $512 \times 512$. The bipolar gradients parameters are Gflow $=6.4$ $\mathrm{G} / \mathrm{cm}$, tflow $=6 \mathrm{~ms}$, resulting in a velocity sensitivity of $\mathrm{K}=1.56$ $\mathrm{s} / \mathrm{mm}$ (maximum velocity encoded without inducing phase 
wrapping $V \max =2 \mathrm{~mm} / \mathrm{s}$ ). Using TE/TR $=44.7 / 3000 \mathrm{~ms}$, the total acquisition time is $12 \mathrm{~min} 48 \mathrm{~s}$ per velocity component $(\mathrm{Vx}, \mathrm{Vy})$. A FORTRAN code was written to obtain a "virtual mask" for the velocity reconstruction and in order to study the partial volume effect due to the imaging method. The experimental data determine the FOV, the resolution and orientation of the microchannel network, and the code outputs a simulated amplitude image where each pixel takes a value from 0 to 1 representing the theoretical water content of the pixel. Since the microchannel network has an average porosity (or liquid volume fraction) of approximately $61.5 \%$, we expect to observe a significant effect of the water/PDMS interface on the signal quality. A close-up of the velocity vectors is shown using an experimental mask (obtained by thresholding an experimental amplitude image) in Figure 4(a), and the same field processed by using our virtual mask are shown in Figure 4(b). The importance of the virtual mask is manifested when computing volumetric flow rates because of the experimental noise and inaccuracies introduced in the experimental mask caused by binary thresholding.
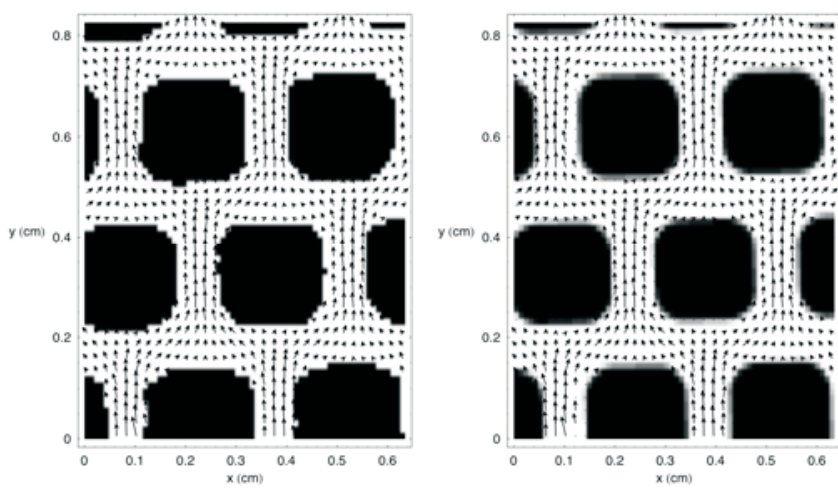

Figure 4. Close-up of velocity vectors in the microchannel network obtained for Re $=1$, using (left panel) an experimental mask (obtained by thresholding an experimental amplitude image), and (right panel) a numerically-obtained "virtual" mask.

Given the spatial heterogeneity of the inlet flow, a readily available validation test of the MRI measurements is to verify that the amount of fluid circulated through the microchannel network is equal to that imposed by the syringe pump, i.e. to check the mass balance throughout the microchannel array. Both microchannel networks of the phantom are flooded and the two-dimensional velocity field is measured for $\mathrm{Re}=1.5(\mathrm{Q}=82.2 \mathrm{ml} / \mathrm{hr}$, Uavg $=$ $0.6 \mathrm{~mm} / \mathrm{s}$ ) with a high spatial resolution: 256 data points are collected in both read-out and phase-encoding direction. The $5 \mathrm{~cm}$ x $6.5 \mathrm{~cm}$ FOV is carefully chosen so that both networks are lined up one on top of the other and an 11-mm-thick slice is used to capture both networks in a single slice. Again, the data matrix is zero-padded to $512 \times 512$. The bipolar gradients parameters are Gflow $=5 \mathrm{G} / \mathrm{cm}$, tflow $=7 \mathrm{~ms}$, resulting in a velocity sensitivity of $\mathrm{K}=1.61 \mathrm{~s} / \mathrm{mm}$ (or a maximum velocity encoded without phase wrapping $\mathrm{Vmax}=1.96 \mathrm{~mm} / \mathrm{s})$. Using $\mathrm{TE} / \mathrm{TR}=49 / 4000 \mathrm{~ms}$, the total acquisition time is $17 \mathrm{~min} 4 \mathrm{~s}$ per velocity component $(\mathrm{Vx}$, Vy). A virtual mask was computed based on the MRI amplitude images and applied to extract the velocity field inside the combined microchannel networks. The corrected volumetric flow rate, Qexp, normal to the (axial) y-direction is computed for each y location, as well as for each repeated cell in the periodic microchannel arrays in the axial direction. We find that Qexp then overestimates the volumetric flow rate $(\mathrm{Q}=82.2 \mathrm{ml} / \mathrm{hr})$ imposed by the syringe pump by $1 \%$ on average. Some deviations occur in regions of the microchannel networks where the flow direction varies from axial (y) to transverse (x) and the cross-sectional area expands by a factor of three. The volumetric flow rate averaged over each repeated cell of the microchannel networks is relatively constant (standard deviation $<3 \%$ of $\mathrm{Q}$ ) indicating that the velocity measurements are self-consistent in that mass is conserved from cell to cell in the axial direction.

\section{CONCLUSIONS}

We report here the first use of MRI for the quantitative visualization of flow through arrays of interconnected microchannels, which are the standard elements in continuousflow microfluidic devices [1-3]. This was accomplished by employing a regular sized volume RF coil, and choosing the phase contrast spin echo MRI protocol (PC-MRI). A systematic comparison of PC-MRI with other available MRI velocimetry techniques revealed the superiority of the former.

The present work supports the view that are several advantages inherent to the use of micro-MRI velocimetry to probe microfluidics. The use of a single platform and experimental setup for the extraction of both topological and velocimetry data is unique to MRI. It is also evident that flow measurements are completely non-invasive (no tracer particles or dyes are required), and that slice selection and penetration into the opaque device by MRI are unconstrained. This is not limited to PDMS, and it simply requires that the magnetic susceptibility gradients between the fluid and the solid material be minimized. MRI-compatible materials include the whole gamut of materials used in the fabrication of complex, multilayered, microfluidic networks [1-3]. In contrast to velocimetry techniques using optical microscopy, the field-of-view afforded by MRI can be arbitrarily oriented in 3-D space and it can encompass the full device, so no microscope stage repositioning is necessary during the imaging. Finally, it is worth mentioning that, by a simple change of imaging protocol, the same MRI set-up can be used to image quantitatively mass transport [23] in microfluidic devices or phantoms of biomedical significance.

\section{ACKNOWLEDGEMENTS}

The authors gratefully acknowledge the financial support by the NSF Center for Nanoscale Chemical-Electrical-MechanicalManufacturing Systems (Nano-CEMMS, award DMI-0328162), and the NSF Science and Technology Center of Advanced Materials for Purification of Water with Systems (The WaterCAMPWS, award CTS-0120978) in the University of Illinois at Urbana-Champaign (UIUC).

\section{REFERENCES}

[1] D. J. Beebe, G. A. Mensing, and G. M. Walker, "Physics and applications of microfluidics in biology," Annu. Rev. Biomed. Eng., 4:261-286 (2002).

[2] H. A. Stone, A. D. Stroock, and A. Ajdari, "Engineering flows in small devices: microfluidics toward a lab-on-a-chip," Annu. Rev. Fluid Mech., 36:381-411 (2004).

[3] M. Shin, K. Matsuda, O. Ishii, H. Terai, M. KaazempurMofrad, J. Borenstein, M. Detmar, and J. P. Vacanti, "Endothelialized networks with a vascular geometry in microfabricated poly(dimethyl siloxane)," Biomed. Microdev., 6:269-278 (2004). 
[4] J. G. Santiago, S. T. Wereley, C. D. Meinhart, D. J. Beebe, and R. J. Adrian, "A particle image velocimetry system for microfluidics," Expts. Fluids, 25:316-319 (1998).

[5] C. W. Xi, D. L. Marks, D. S. Parikh, L. Raskin, and S. A. Boppart, "Structural and functional imaging of 3D microfluidic mixers using optical coherence tomography," Proc. Natl Acad. Sci. USA, 101:7516-7521 (2004).

[6] D. Liu, S. V. Garimella, and S. T. Wereley, "Infrared microparticle image velocimetry in silicon-based microdevices," Expts. Fluids, 38:385-392 (2005).

[7] S. Devasenathipathy, J. G. Santiago, and K. Takehara, "Particle tracking techniques for electrokinetic microchannel flows," Anal. Chem., 74:3704-3713 (2002).

[8] D. Sinton, "Microscale flow visualization," Microfluid. Nanofluid., 1:2-21 (2004).

[9] U. Tallarek, T. W. J. Scheenen, P. A. de Jager, and H. Van As, "Using NMR displacement imaging to characterize electroosmotic flow in porous media," Magn. Reson. Imaging, 19:453-456 (2001).

[10] X. Zhang and A. G. Webb, "Magnetic resonance microimaging and numerical simulations of velocity fields inside enlarged flow cells used for coupled NMR microseparations," Anal. Chem., 77:1338-1344 (2005).

[11] M. D. Shattuck, R. P. Behringer, G. A. Johnson, and J. G. Georgiadis, "Magnetic resonance imaging of interstitial velocity distributions in porous media," ASME FED, 125:39-45 (1991).

[12] M. D. Shattuck, R. P. Behringer, G. A. Johnson, and J. G. Georgiadis," "Convection and flow in porous media. 1. Visualization by magnetic resonance imaging," J. Fluid Mech., 332: 215-245 (1997).

[13] M. Deurer, I. Vogeler, A. Khrapitchev, and D. Scotter, "Imaging of water flow in porous media my magnetic resonance imaging microscopy," J. Environ. Quality, 31: 487-493 (2002)
[14] X. Ren, S. Staph, and B. Bl"umich, "NMR velocimetry of flow in model fixed-bed reactors of low aspect ratio," AIChE J., 51: 392-405 (2005)

[15] B. Bijeljic, M. D. Mantle, A. J. Sederman, L. F. Gladden, and T. D. Papathanasiou, "Slow flow across macroscopically rectangular fiber lattices and an open region: visualization by magnetic resonance imaging," Phys. Fluids, 13:3652-3663 (2001).

[16] K. W. Moser, "Quantitative measurement of velocity and dispersion via Magnetic Resonance Imaging," Ph.D. dissertation, University of Illinois at Urbana-Champaign (2001).

[17] C. Pozrikidis, Introduction to Theoretical and Computational Fluid Dynamics, Oxford, NY, Oxford Univ. Press (1997).

[18] K. W. Moser, E. C. Kutter, J. G. Georgiadis, R. O. Buckius, H. D. Morris, and J. R. Torczynski, "Velocity measurements of flow through a step stenosis using Magnetic Resonance Imaging," Expts. Fluids, 29:438-447 (2000).

[19] J.D. Seymour, B. Manz, P. T. Callaghan, "Pulsed gradient spin echo nuclear magnetic resonance measurements of hydrodynamic instabilities with coherent structure: Taylor vortices," Phys. Fluids, 11:1104-1113 (1999).

[20] T. J. Mosher and M.B. Smith, "A DANTE tagging sequence for the evaluation of translational sample motion". Magn. Reson. Med. 15: 334-339 (1990).

[21] L. G. Raguin, D. Karampinos, J. G. Georgiadis, and L. Ciobanu, "Comparison of MR Velocimetry Protocols for Microchannels", 47th Experimental Nuclear Magnetic Resonance Conference (ENC), Pacific Grove, CA, USA, April 23 - 28 (2006).

[22] B. H. Jo, L. M. Van Lerberghe, K. M. Motsegood, and D. J. Beebe, "Three-dimensional microchannel fabrication in polydimethylsiloxane (PDMS) elastomer," J. Microelectromech. Syst., 9:76-81 (2000).

[23] L. G. Raguin, D. Hernando, D. Karampinos, L. Ciobanu, B. P. Sutton, Z.-P. Liang, and J. G. Georgiadis, "Quantitative analysis of q-space data," in Proceedings of the IFMBE, 3rd European Medical \& Biological Engineering Conference, Prague, vol. 11, (2005). 